\section{Finger-Prick Blood Sampling for Therapeutic Drug Monitoring: Be Aware of Skin Contamination by Nebulized Drugs}

\section{To the Editor:}

Over the past few years, the number of drugs for inhalation therapy and nebulizer devices has rapidly expanded. ${ }^{1}$ Systemic exposure of inhalational drugs is not only estimated during drug development, but also used to monitor the effects of drug therapy, including toxicity. New liquid chromatography-mass spectrometry techniques have facilitated therapeutic drug monitoring using minimal blood volumes and dried blood spot methods taken by finger-prick sampling.

We recently performed a pharmacokinetic study in children receiving intravenously administered salbutamol for severe acute asthma. ${ }^{2}$ During data analysis, we noticed 8 aberrantly high concentrations of salbutamol (Fig. 1) that could not be attributed to the pharmacokinetic profile of the drug, drug administration errors, samples taken from the drug administration line, clinical symptoms, sample mix-ups, or other logistic errors. All children were switched back to salbutamol nebulization when the intravenous administration was sufficiently reduced or stopped. An alternative explanation was contamination of the finger-prick sample due to drug transfer from the skin to the blood sample in the microtainer. Salbutamol may be present on the fingers as a consequence of holding the nebulizing device and the subsequent touching of the mouth or the inner side of the device during therapy. This contamination may result in the overestimation of the true blood concentration. ${ }^{3}$ Totally, 9 samples (7\%) were obtained through a finger prick after switching back to nebulization, and 6 of these samples were found to have

The authors declare no conflict of interest. aberrantly high drug concentrations. Samples during nebulization before the start of intravenous therapy were all taken from an arterial line or central venous catheter, instead of using a finger prick.

As our pharmacy department performs regular therapeutic drug monitoring for inhalational drugs, potential drug contamination of the skin may seriously impact interpretation of results and patient care, for other drugs also. We therefore designed a quality improvement project that tested the hypothesis that nebulized drugs may contaminate a patient's fingers, leading to false high blood concentrations from finger-prick samples. The medical ethical review board granted a waiver after reviewing the protocol. In addition, the healthy volunteers provided written informed consent.

\section{METHODS}

We performed 2 experiments: the first was to examine proof-of-concept, and the second was to investigate the effects of mask holding and 2 cleaning methods.

For the first experiment, a research nurse prepared the salbutamol nebulization equipment in accordance with the protocol used in clinical care: $2-\mathrm{mL}$ $\mathrm{NaCl} 0.9 \%$ and $2-\mathrm{mL}$ salbutamol $2 \mathrm{mg} /$ $\mathrm{mL}$ were combined in the ICU nebulizing system (Hudson ACI, Paediatric aerosol mask combined with Hudson Micro-Mist nebulizer). The research nurse turned on the airflow. The prepared salbutamol solution was nebulized onto the hand of a salbutamol-free adult volunteer. The hand was placed directly at the opening of the nebulizing mask. This volunteer was not involved in the preparation of the nebulizer set-up and did not touch the salbutamol solution. After approximately 5 minutes, a fingerprick sample of approximately $0.5 \mathrm{~mL}$ was taken from the sprayed hand into a microtainer.

The second experiment was performed in 2 healthy adult volunteers. The first volunteer held the nebulizer in their left hand at the bottom, close to the salbutamol reservoir, in the same manner as older children. The second volunteer held the nebulizer with their left hand at the mask, in the same manner as younger children. Both volunteers sprayed the salbutamol from a distance of $10 \mathrm{~cm}$ onto their right hand over a period of 5 minutes. On their right hand, one finger was not cleaned, one finger was swabbed with alcohol to mimic standard disinfection before blood sampling, and one finger was thoroughly cleaned with water and soap. A finger-prick blood sample was taken from each of these 3 fingers in addition to one finger-prick sample from the left hand holding the mask. All plasma samples were stored at $-80^{\circ} \mathrm{C}$ until analysis using a validated liquid chromatography-mass spectrometry method.

\section{RESULTS}

In the first experiment, spraying salbutamol over the hand of the volunteer resulted in concentrations of $>1000$ $\mathrm{mcg} / \mathrm{L}$ R-salbutamol and $>1000 \mathrm{mcg} / \mathrm{L}$ S-salbutamol. This was much higher than the range of concentrations analyzed during intravenous salbutamol infusion in children included in the trial (R-salbutamol: median, $66 \mathrm{mcg} / \mathrm{L}$; S-salbutamol: median, $106 \mathrm{mcg} / \mathrm{L}$ ), but within the range of concentrations found in the 8 outlier samples in the pilot study (R-salbutamol range: $504-1918 \mathrm{mcg} / \mathrm{L}$; S-salbutamol range: $636-2018 \mathrm{mcg} / \mathrm{L}$ ).

In the second experiment, the concentrations measured on the uncleaned finger were 686 and $639 \mathrm{mcg} / \mathrm{L}$, respectively, for one volunteer, and 82 and $75 \mathrm{mcg} / \mathrm{L}$ for the other volunteer, respectively, for R-salbutamol and S-salbutamol. Cleaning with alcohol wipes resulted in $39 \%-56 \%$ lower salbutamol concentrations for both volunteers (398 and 36 $\mathrm{mcg} / \mathrm{L}$ R-salbutamol, 389 and $34 \mathrm{mcg} / \mathrm{L}$ S-salbutamol). Cleaning with water and soap decreased the concentrations by $94 \%-100 \% \quad(30$ and $5 \mathrm{mcg} / \mathrm{L}$ R-salbutamol, respectively; $29 \mathrm{mcg} / \mathrm{L}$ and $<2 \mathrm{mcg} / \mathrm{L}$ S-salbutamol, respectively). The concentrations measured on the finger of the left hand were 8 $\mathrm{mcg} / \mathrm{L}$ for R-salbutamol and $10 \mathrm{mcg} / \mathrm{L}$ for S-salbutamol for the volunteer holding the system at the reservoir and 78 $\mathrm{mcg} / \mathrm{L}$ for R-salbutamol and $56 \mathrm{mcg} / \mathrm{L}$ 

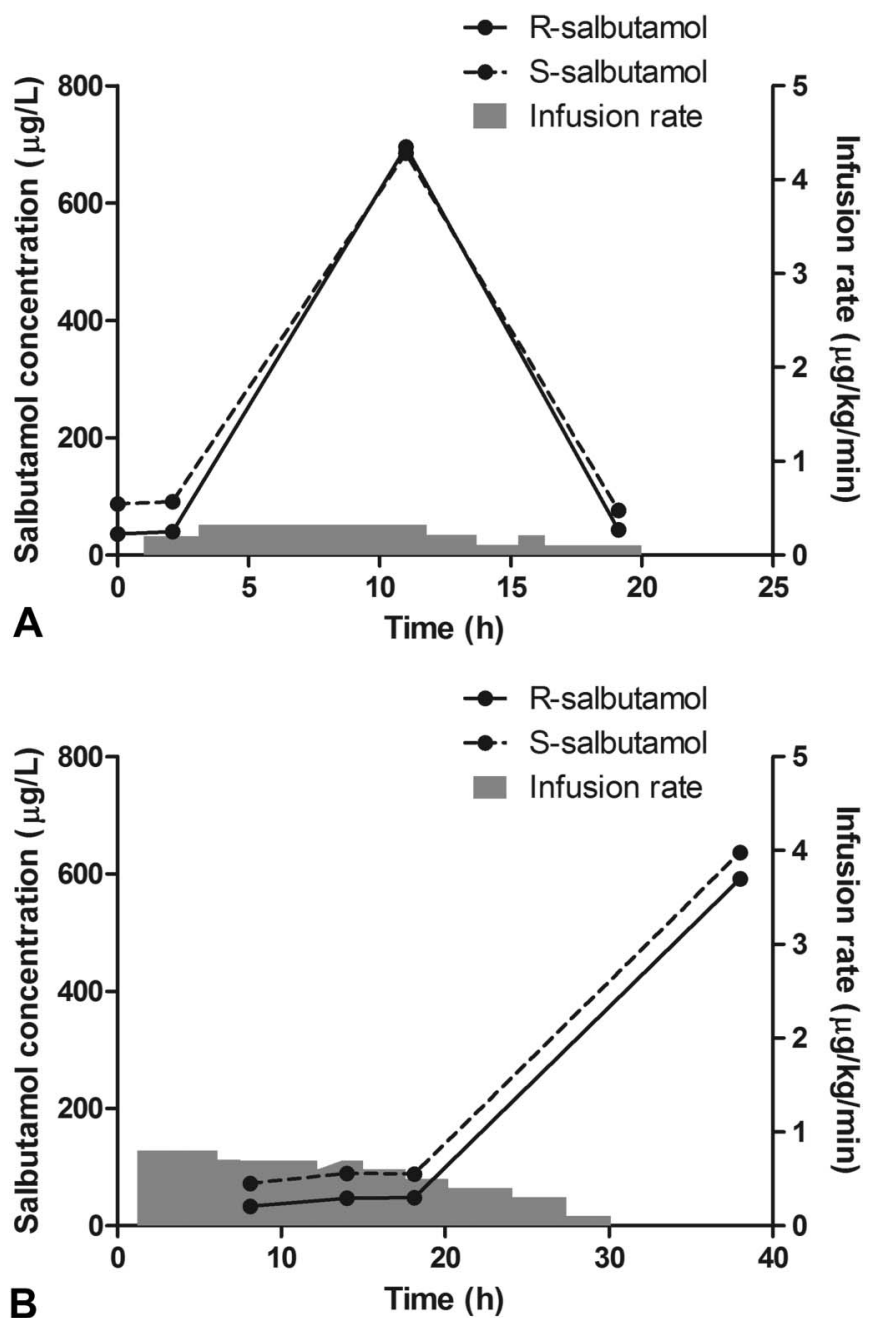

FIGURE 1. Concentration-time profiles, including infusion rate for 2 patients in which aberrantly high concentrations of salbutamol, were analyzed. The measured Rsalbutamol and S-salbutamol concentrations and infusion rates are presented in the figure. Patient A restarted nebulization at 10 hours after inclusion, and patient $B$ restarted nebulization at 18 hours after inclusion.

for S-salbutamol for the volunteer holding the system at the mask.

\section{CONCLUSIONS}

After nebulizing, skin contamination can result in falsely elevated plasma concentrations in finger-prick blood samples. The contamination is probably lesser on the hand holding the system at the reservoir than the mask; however, the fingers are still contaminated. The extreme high concentrations may be the result of a longer nebulizing period, or the child touching the mouth or the inner side of the device. We expect this contamination is comparable in new dried-blood spot techniques using finger-prick sampling. Finger-prick blood sampling should be used cautiously in therapeutic drug monitoring and pharmacokinetic studies of nebulized drugs. To overcome this problem, blood sampling practices for these drugs should be carefully considered to prevent elevated levels and corresponding biased conclusions. As a precaution, wearing gloves on the sampling hand or traditional venipuncture (if the sampling place is covered during inhalation) should be considered. Cleaning techniques using both alcohol wipes or water and soap result in unpredictable contamination.

Brenda C. M. de Winter, PhD* Matthijs de Hoog, MD $\uparrow$ Nienke J. Vet, PhD $\dagger$ Joke H. Dunk-Craaijo $\dagger$ Birgit C. P. Koch, PhD* Saskia N. de Wildt, MD, PhD $\dagger, \ddagger$ *Department of Hospital Pharmacy, Erasmus University Medical Center, Rotterdam, the Netherlands $\dagger$ Intensive Care and Department of Pediatric Surgery, Erasmus MC-Sophia Children's Hospital, Rotterdam, the Netherlands tDepartment of Pharmacology and Toxicology, Radboud University, Nijmegen, the Netherlands

\section{REFERENCES}

1. Martin AR, Finlay WH. Nebulizers for drug delivery to the lungs. Expert Opin Drug Deliv. 2015;12:889-900.

2. Vet $\mathrm{N}$, de Winter BCM, de Wildt $\mathrm{SN}$, et al. Population pharmacokinetics of intravenous salbutamol in children with refractory status asthmaticus. Clin Pharmacokinet. 2019;59: 257-264.

3. Redmann S, Wainwright C, Stacey S, et al. Misleading high tobramycin plasma concentrations can be caused by skin contamination of fingerprick blood following inhalation of nebulized tobramycin (TOBI): a short report. Ther Drug Monit. 2005;27:205-207. 